\title{
PAIRWISE INCOMPATIBLE GENERIC FAMILIES
}

\author{
WOJCIECH GUZICKI
}

(Communicated by Andreas R. Blass)

\begin{abstract}
Let $M$ be a countable model of $\mathbf{Z F}^{-}$. There exists a family $\mathscr{F}$ of $2^{2^{\omega}}$ models of $\mathbf{Z F}^{-}$each obtained from $M$ by adjoining an $M$-generic family of $2^{\omega}$ Cohen reals, such that no two distinct models in $\mathscr{F}$ have a common extension to a model of $\mathbf{Z F}^{-}$with the same ordinals.
\end{abstract}

\section{INTRODUCTION}

In his paper [3] A. Mostowski showed that for any countable model $M$ of Gödel-Bernays set theory there exists a family $\mathscr{F}$ of models of $G B$ with the following properties:

(1) all models in $\mathscr{F}$ are extensions of $M$ with the same universe of sets;

(2) no two different models in $\mathscr{F}$ have a common extension to a model of $G B$ with the same ordinals;

(3) all models in $\mathscr{F}$ have power $\omega_{1}$; and

(4) the power of $\mathscr{F}$ is $2^{\omega_{1}}$.

A. Mostowski conjectured that Martin's Axiom implies the existence of a similar family of power $2^{2^{\omega}}$ consisting of models of power $2^{\omega}$. In [2] M. Kaufmann proved without any additional assumptions that such a family of power $2^{2^{\omega}}$ does exist. Both proofs were based on a construction of generic $M$-classes $G_{1}, G_{2} \subseteq O n$ such that the intersection $G_{1} \cap G_{2}$ is a cofinal subset of $O n \cap M$ of order type $\omega$. Each extension of $M$ was generated by a certain family of generic $M$-classes. The families were constructed in such a way that any two of them contained a pair of generic classes with the above property.

Another construction of "fat and short" models of set theory is given in [1]. In that paper $\mathrm{H}$. Friedman gives a definition of a generic family of Cohen reals and proved the existence of generic families of power $2^{\omega}$ (see Definition 1.1 and Lemma 1.2 below). He also showed that every generic family generates a model of $\mathbf{Z F}$. A simple modification of his proof gives a construction of models of $\mathbf{Z F C}^{-}$and second order arithmetic generated by such families.

Received by the editors June 24, 1988 and, in revised form, October 24, 1989.

1980 Mathematics Subject Classification (1985 Revision). Primary 03C62; Secondary 03E25. 
In this paper we introduce the notion of incompatibility of Friedman's generic families (Definition 4.2). A natural question arises of whether there exist $2^{2^{\omega}}$ pairwise incompatible generic families of Cohen reals which would extend Kaufmann's result to $\mathbf{Z F}, \mathbf{Z F C}{ }^{-}$, or $A_{2}$. The construction of M. Kaufmann doesn't give an immediate generalization.

In the present paper we construct $2^{2^{\omega}}$ pairwise incompatible generic families of Cohen reals (Corollary 4.4). The results of $\S 1$ are due to H. Friedman, Lemma 3.2 to R. Solovay.

\section{GeNERIC FAMILIES}

We consider the usual Cohen's notion of forcing $\mathbb{P}$. For any family $\mathscr{D}$ of dense subsets of $\mathbb{P}$ (or $\mathbb{P}^{n}$ ) we define the notion of $\mathscr{D}$-generic filters in $\mathbb{P}$ (resp. in $\mathbb{P}^{n}$ ) in the usual way. We shall always assume that the family $\mathscr{D}$ contains dense subsets which guarantee that the union of a generic filter is a total function from $\omega$ into $\{0,1\}$. This allows us to identify a generic filter $G$ with the Cohen real $\{n \in \omega:(\bigcup G)(n)=0\}$.

Let us assume now that for any $n \in \omega$ we are given a family $\mathscr{D}_{n}$ of dense subsets of $\mathbb{P}^{n}$. We consider the sequence $\mathscr{D}=\left\langle\mathscr{D}_{n}: n \in \omega\right\rangle$.

Definition 1.1. A family $\mathscr{G}$ of filters in $\mathbb{P}$ is weakly $\mathscr{D}$-generic iff it is infinite and for any $n \in \omega$ and any $G_{1}, \ldots, G_{n} \in \mathscr{G}$ the sequence $\left\langle G_{1}, \ldots, G_{n}\right\rangle$ is $\mathscr{D}_{n}$-generic in $\mathbb{P}^{n}$. The family $\mathscr{G}$ is $\mathscr{D}$-generic iff it is weakly $\mathscr{D}$-generic and for any $\rho \in \mathbb{P}$ there exists $G \in \mathscr{G}$ such that $\rho \in G$.

If $M$ is a model of $\mathbf{Z F}^{-}$then a family $\mathscr{G}$ is $M$-generic iff it is $\mathscr{D}$-generic, where $\mathscr{D}=\left\langle\mathscr{D}_{n}: n \in \omega\right\rangle$ and $\mathscr{D}_{n}$ is the family of dense subsets of $\mathbb{P}^{n}$ which belong to $M$.

Lemma 1.2. If $\mathscr{D}$ is a sequence of countable families of dense subsets of the notions of forcing $\mathbb{P}^{n}$ then there exists a weakly $\mathscr{D}$-generic family $\mathscr{G}$ of power $2^{\omega}$.

Proof. Let $S=\bigcup_{n \in \omega} 2^{n}$. We define a function $f: S \rightarrow \mathbb{P}$ so that the following conditions hold:

(1) $f(0)=0$;

(2) $f$ is one-to-one;

(3) $s_{1} \subseteq s_{2} \rightarrow f\left(s_{1}\right) \subseteq f\left(s_{2}\right)$ for any $s_{1}, s_{2} \in S$;

(4) $s_{1} \cup s_{2} \notin S \rightarrow f\left(s_{1}\right) \cup f\left(s_{2}\right) \notin \mathbb{P}$ for any $s_{1}, s_{2} \in S$;

(5) for any $n \in \omega$ and any $D \in \mathscr{D}_{n}$ there exists $m \in \omega$ such that for any distinct finite sequences $s_{1}, \ldots, s_{n} \in 2^{m}$ there exist conditions $\rho_{1}, \ldots, \rho_{n} \in \mathbb{P}$ such that:

$$
\left\langle\rho_{1}, \ldots, \rho_{n}\right\rangle \in D \text { and }\left\langle f\left(s_{1}\right), \ldots, f\left(s_{n}\right)\right\rangle \leq\left\langle\rho_{1}, \ldots, \rho_{n}\right\rangle .
$$

The details of the construction are left for the reader. Now for $h: \omega \rightarrow 2$ we define the set $G_{h}=\{\rho \in \mathbb{P}: \exists m \in \omega[f(h \mid m) \leq \rho]\}$ and easily find that the family $\mathscr{G}=\left\{G_{h}: h \in 2^{\omega}\right\}$ is the required weakly $\mathscr{D}$-generic family. 


\section{AUTOMORPHISMS}

If $\tau_{1}, \ldots, \tau_{n}$ are automorphisms of $\mathbb{P}$ then by $\tau_{1} \times \cdots \times \tau_{n}$ we denote an automorphism of $\mathbb{P}^{n}$ defined as follows:

$$
\tau_{1} \times \cdots \times \tau_{n}\left(\left\langle\rho_{1}, \ldots, \rho_{n}\right)\right\rangle=\left\langle\tau_{1}\left(\rho_{1}\right), \ldots, \tau_{n}\left(\rho_{n}\right)\right\rangle .
$$

Definition 2.1. For any $a \subseteq \omega$ we define an automorphism $\tau_{a}: \mathbb{P} \rightarrow \mathbb{P}$ with the formula:

$$
\tau_{a}(\rho)(n)= \begin{cases}\rho(n) & \text { for } n \notin a, \\ 1-\rho(n) & \text { for } n \in a .\end{cases}
$$

We easily notice that for any $a, b \subseteq \omega$ we have $\tau_{a} \circ \tau_{b}=\tau_{a \Delta b}$. Thus automorphisms $\tau_{a}$ and $\tau_{b}$ commute: $\tau_{a} \circ \tau_{b}=\tau_{b} \circ \tau_{a}$.

If $a_{1}, \ldots, a_{n} \subseteq \omega$ are finite then the automorphism $\tau_{a_{1}} \times \cdots \times \tau_{a_{n}}: \mathbb{P}^{n} \rightarrow \mathbb{P}^{n}$ will be called a finite modification. A sequence $\mathscr{D}=\left\langle\mathscr{D}_{n}: n \in \omega\right\rangle$ of dense subsets of the notions of forcing $\mathbb{P}^{n}$ is called closed under finite modifications iff for any finite modification $\tau_{a_{1}} \times \cdots \times \tau_{a_{n}}$ and any $D \in \mathscr{D}_{n}$ the set $\tau_{a_{1}} \times \cdots \times \tau_{a_{n}}(D)$ belongs to $\mathscr{D}_{n}$ as well.

Let us assume now that $\mathscr{G}$ is a weakly $\mathscr{D}$-generic family and that $\mathscr{D}$ is closed under finite modifications. We choose filters $G_{1}, \ldots, G_{n}, \ldots$ from $\mathscr{G}$ and find finite sets $a_{1}, \ldots, a_{n}, \ldots$ so that the filters $\tau_{a_{1}}\left(G_{1}\right), \ldots, \tau_{a_{n}}\left(G_{n}\right), \ldots$ extend all conditions from $\mathbb{P}$. Then the family $\mathscr{G}^{*}$ obtained from $\mathscr{G}$ by replacing filters $G_{1}, \ldots, G_{n}, \ldots$ with the filters $\tau_{a_{1}}\left(G_{1}\right), \ldots, \tau_{a_{n}}\left(G_{n}\right), \ldots$ is $\mathscr{D}$-generic. Together with Lemma 1.2 we obtain the following.

Corollary 2.3. If a sequence $\mathscr{D}=\left\langle\mathscr{D}_{n}: n \in \omega\right\rangle$ of countable families of dense subsets of the notions of forcing $\mathbb{P}^{n}$ is closed under finite modifications then there exists a $\mathscr{D}$-generic family $\mathscr{G}$ of power $2^{\omega}$.

Now let $a \subseteq \omega$. We define $\tau_{a}^{0}=$ Id and $\tau_{a}^{1}=\tau_{a}$. Next, for any finite sequence $\left\langle\varepsilon_{1}, \ldots, \varepsilon_{n}\right\rangle \in 2^{n}$ we define

$$
\tau_{a}^{\varepsilon_{1}, \ldots, \varepsilon_{n}}=\tau_{a}^{\varepsilon_{1}} \times \cdots \times \tau_{a}^{\varepsilon_{n}}
$$

and

$$
T_{n}=\left\{\tau_{a}^{\varepsilon_{1}, \ldots, \varepsilon_{n}}:\left\langle\varepsilon_{1}, \ldots, \varepsilon_{n}\right\rangle \in 2^{n}\right\}
$$

Next, for any family $\mathscr{D}_{n}$ of dense subsets of $\mathbb{P}^{n}$ we define

$$
a\left(n, \mathscr{D}_{n}\right)=\bigcup_{n \in \omega} \tau\left(\mathscr{D}_{n}\right)
$$

and finally for any sequence $\mathscr{D}=\left\langle\mathscr{D}_{n}: n \in \omega\right\rangle$ of families of dense subsets of the notions of forcing $\mathbb{P}^{n}$ we define

$$
a(\mathscr{D})=\left\langle a\left(n, \mathscr{D}_{n}\right): n \in \omega\right\rangle \text {. }
$$

From the commutativity of automorphisms of form $\tau_{a}$ it easily follows

Lemma 2.3. If a sequence $\mathscr{D}$ is closed under finite modifications then so is the sequence $a(\mathscr{D})$ for any $a \subseteq \omega$. 


\section{3. $a$-DEPENDENT GENERIC REALS AND FAMILIES}

We begin with the following

Definition 3.1. Let $a \subseteq \omega$. We say that $\mathscr{D}$-generic filters $G_{1}, G_{2} \subseteq \mathbb{P}$ are $a$-dependent iff the set

$$
a \triangle\left\{n \in \omega:\left(\bigcup G_{1}\right)(n) \neq\left(\bigcup G_{2}\right)(n)\right\}
$$

is finite.

Lemma 3.2. For any $a \subseteq \omega$ and any countable family $\mathscr{D}$ of dense subsets of $\mathbb{P}$ there exists a-dependent $\mathscr{D}$-generic filters $G_{1}, G_{2} \subseteq \mathbb{P}$.

Proof. We consider automorphisms $\tau_{a}$ and the family $\mathscr{D} \cup \tau_{a}(\mathscr{D})$ of dense subsets of $\mathbb{P}$. We take a $\left(\mathscr{D} \cup \tau_{a}(\mathscr{D})\right)$-generic filter $G_{1}$ and define $G_{2}=\tau_{a}\left(G_{1}\right)$. We easily see that $G_{1}$ and $G_{2}$ are $\mathscr{D}$-generic and $a=\left\{n \in \omega:\left(\bigcup G_{1}\right)(n) \neq\right.$ $\left.\left(\bigcup G_{2}\right)(n)\right\}$.

Definition 3.3. Two $\mathscr{D}$-generic (or weakly $\mathscr{D}$-generic) families $\mathscr{G}_{1}$ and $\mathscr{G}_{2}$ are $a$-dependent iff there exist $a$-dependent filters $G_{1} \in \mathscr{G}_{1}$ and $G_{2} \in \mathscr{G}_{2}$.

Theorem 3.4. If $\mathscr{D}$ is a sequence of countable families of dense subsets of the notions of forcing $\mathbb{P}^{n}$ closed under finite modifications then for any $a \subseteq \omega$ there exists a set of power $2^{2^{\omega}}$ of a-dependent $\mathscr{D}$-generic families of power $2^{\omega}$.

Proof. We consider a sequence $a(\mathscr{D})$. By Corollary 2.2 there exists an $a(\mathscr{D})$ generic family $\mathscr{G}=\left\{G_{f}: f \in 2^{\omega}\right\}$ of power $2^{\omega}$. Now we define $G_{f}^{0}=G_{f}$ and $G_{f}^{1}=\tau_{a}\left(G_{f}\right)$. For any function $h: 2^{\omega} \rightarrow 2$ we put $\mathscr{G}_{h}=\left\{G_{f}^{h(f)}: f \in 2^{\omega}\right\}$. From the proof of Lemma 3.2 it follows that for different $h_{1}$ and $h_{2}$ the families $\mathscr{G}_{h_{1}}$ and $\mathscr{G}_{h_{2}}$ are $a$-dependent. We show now that the families $\mathscr{G}_{h}$ are weakly $\mathscr{D}_{\text {- }}$ generic.

Let $G_{f_{1}}^{h\left(f_{1}\right)}, \ldots, G_{f_{n}}^{h\left(f_{n}\right)} \in \mathscr{G}_{h}$. Then

$$
\left\langle G_{f_{1}}^{h\left(f_{1}\right)}, \ldots, G_{f_{n}}^{h\left(f_{n}\right)}\right\rangle=\tau_{a}^{h\left(f_{1}\right), \ldots, h\left(f_{n}\right)}\left(\left\langle G_{f_{1}}, \ldots, G_{f_{n}}\right\rangle\right) .
$$

Since $\left\langle G_{f_{1}}^{h\left(f_{1}\right)}, \ldots, G_{f_{n}}^{h\left(f_{n}\right)}\right\rangle$ is $a(\mathscr{D})$-generic, we see that $\left\langle G_{f_{1}}^{h\left(f_{1}\right)}, \ldots, G_{f_{n}}^{h\left(f_{n}\right)}\right\rangle$ is $\mathscr{D}$-generic.

Finally, since the sequence $\mathscr{D}$ is closed under finite modifications, each family $\mathscr{G}_{h}$ can be modified as in $\S 2$ so that it becomes $\mathscr{D}$-generic. Since the filters in families $\mathscr{G}_{h}$ are at most finitely modified, the constructed generic families remain $a$-dependent.

\section{INCOMPATIBLE GENERIC FAMILIES}

Let $M$ be a countable transitive model of $\mathbf{Z F}^{-}$.

Definition 4.1. Two $M$-generic Cohen reals $G_{1}, G_{2} \subseteq \mathbb{P}$ are incompatible iff there is no model $N$ of $\mathbf{Z F}^{-}$such that $M \subseteq N, O n \cap M=O n \cap N$, and $G_{1}, G_{2} \in N$. 
Definition 4.2. Two $M$-generic families $\mathscr{G}_{1}$ and $\mathscr{G}_{2}$ are incompatible iff there exist incompatible Cohen generic reals $G_{1} \in \mathscr{G}_{1}$ and $G_{2} \in \mathscr{G}_{2}$.

Theorem 4.3. There exists a set of power $2^{2^{\omega}}$ of pairwise incompatible M-generic families of power $2^{\omega}$.

Proof. We take a set $a \subseteq \omega$ which does not belong to any model of $\mathbf{Z F}^{-}$of the same height as $M$ (e.g. $a$ can code a well-ordering of $\omega$ of type $O n \cap M$ ). Now it is enough to observe that any $a$-dependent generic families are incompatible and to apply Theorem 3.4.

Corollary 4.4. For any countable model $M$ of $\mathbf{Z F}^{-}$there exists a family $\mathscr{F}$ of models of $\mathbf{Z F}^{-}$extending $M$ such that

(1) no two different models in $\mathscr{F}$ have a common extension to a model of $\mathbf{Z F}^{-}$with the same ordinals,

(2) all models in $\mathscr{F}$ have power $2^{\omega}$,

(3) the power of $\mathscr{F}$ is $2^{2^{\omega}}$.

We can also observe that the above results and proofs can easily be generalized to the case of generic sets of higher power or to the case of generic classes, thus giving theorems of Mostowski and Kaufmann.

\section{REFERENCES}

1. H. Friedman, Large models of countable height, Trans. Amer. Math. Soc. 201 (1975), 227-239.

2. M. Kaufmann, Mutually generic classes and incompatible extensions, Fund. Math. 121 (1984), 213-218.

3. A. Mostowski, A remark on models of Gödel-Bernays axioms for set theory sets and classes, Studies in Logic and the Foundations of Mathematics (G. H. Müller, ed.), North-Holland, 1976, pp. 325-340.

Institute of Mathematics, University of Warsaw, PKiN, 00-901 Warszawa, Poland 